ARTículo

\title{
Tensiones epistemológicas en la implementación de la Educación Intercultural Bilingüe*
}

Katerin Arias-Ortega a

Segundo Quintriqueo ${ }^{b}$

\section{Resumen}

El artículo busca indagar las tensiones epistemológicas existentes entre el profesor mentor y el educador tradicional (sabio mapuche), en la implementación de la Educación Intercultural Bilingüe (EIB) en tres escuelas de La Araucanía, Chile. La metodología utilizada es la investigación cualitativa, a través de la aplicación de entrevistas semi-dirigidas a profesores mentores y educadores tradicionales encargados de implementar la EIB. Los principales resultados dan cuenta de que existe una relación educativa de carácter colonial del profesor mentor hacia el educador tradicional, lo que limita la implementación de la EIB. Estas tensiones están asociados a prácticas de minorización, asimetría y hegemonía del conocimiento escolar de raíz eurocéntrico occidental sobre la Enseñanza de la lengua y la cultura mapuche.

Palabras clave: Relación educativa. Educación colonial. Educación intercultural.

\section{Introducción}

La literatura normativa impulsada por el Ministerio de Educación de Chile (Mineduc), desde la década de 1990 sobre la Educación Intercultural Bilingüe (EIB), actualmente plantea el desafío de evaluar su implementación en el aula (VALDEBENITO, 2017). Esta política sienta sus bases en la promulgación de la Ley Indígena $n^{\circ} 19.253$ de 1993, que establece las normas sobre protección, fomento y desarrollo de los pueblos indígenas en Chile, mediante una Educación que fomente su historia, lengua y cultura propia. Asimismo, a nivel internacional,

\footnotetext{
* Agradecimientos a los proyectos PROFONDECYT 2019PF-KA-05, financiado por la Vicerrectoría de Investigación y Posgrado de la Universidad Católica de Temuco. Al proyecto FONDECYT INICIACIÓN 11200306 y FONDECYT REGULAR 1181531.

a Universidad Católica de Temuco, La Araucanía, Chile.

b Universidad Católica de Temuco, La Araucanía, Chile.
}

Recibido en: 22 mar. 2019

Aceptado en: 30 set. 2020 
la EIB se enmarca en el Convenio 169 de la Organización Internacional del Trabajo (OIT) sobre pueblos indígenas y tribales de países independientes, ratificado por Chile en el año 2009. La Educación intercultural como política pública también se enmarca en la Declaración de las Naciones Unidas sobre los Derechos Universales de Pueblos Indígenas, que establece el derecho a fomentar $\mathrm{y}$ transmitir a las nuevas generaciones su historia, tradiciones orales y su propia lengua, mediante una Educación contextualizada a su propio marco social y cultural. En este sentido, la implementación de la EIB históricamente se ha sustentado desde un enfoque funcional a las necesidades del Estado, mediante lineamientos estandarizados, centralistas y homogéneos que invisibilizan la episteme indígena en las diferentes territorialidades mapuches (QUINTRIQUEO; ARIAS-ORTEGA, 2019). Estas problemáticas limitan la implementación de la EIB y, por ende, inciden negativamente en la incorporación de saberes y conocimientos educativos para su articulación con los conocimientos escolares en las distintas asignaturas del currículum escolar.

En ese contexto, el problema que aborda el artículo se relaciona con la política educativa del Mineduc, que desde el año 1996 implementa la modalidad educativa de EIB para visibilizar los conocimientos indígenas en la Educación escolar (CHILE, 2010). Sin embargo, en la realidad empírica observamos que el trabajo pedagógico en el aula, para enseñar la lengua y la cultura mapuche en la implementación de la EIB, se realiza desde una relación educativa asimétrica entre el profesor mentor, el educador tradicional y los estudiantes, donde persiste una práctica de invisibilización de los saberes y conocimientos educativos mapuches (ARIAS-ORTEGA, 2019). Al mismo tiempo, desde una perspectiva teórica, constatamos que la escuela por ser de carácter monocultural eurocéntrica occidental, desconoce los conocimientos indígenas. En ese contexto, sostenemos que la implementación de la política educativa sobre EIB está limitada por una relación educativa tensionada epistemológicamente entre los agentes educativos que la desarrollan.

El objetivo del artículo es identificar las tensiones epistemológicas que existen entre el profesor mentor y educador tradicional en tanto agentes educativos encargados de implementar la política educativa sobre EIB en La Araucanía, Chile.

\section{Educación Intercultural arraigada a un enfoque educativo colonial}

La política de Educación intercultural surge tanto en el contexto europeo como latinoamericano, especialmente desde la época colonial, donde las primeras 
relaciones interculturales entre colonizadores y colonizados dan cuenta de una relación intercultural (BISHOP, 2010; DIETZ; MATEOS CORTÈS, 2011; PALADINO; CZARNI, 2012). Dichas relaciones interculturales se constituyen desde un ámbito social, económico e institucional, en los procesos de conquista y colonización. En el ámbito social fue producto del contacto con los españoles en el proceso de evangelización para civilizar a los indígenas mediante la enseñanza de la lengua dominante y la escritura en desmedro de la oralidad (SERRANO; PONCE DE LEÓN; RENGIFO, 2012). En el ámbito económico se observó tanto en las Américas como en Chile el intercambio en la economía, específicamente en La Araucanía, asociado a la ganadería, la producción textil, la alfarería y agricultura (BENGOA, 2008). En el ámbito institucional surgió como una respuesta de los Estados a la Educación escolar que demandan los pueblos indígenas, desde principios del siglo pasado (TURRA DÍAZ, 2016). En efecto, los espacios de relaciones interculturales entre indígenas y no indígenas, tanto en el plano social como económico e institucional, han generado procesos de transformación en contexto indígena y/o colectividades subalternizadas (NOVARO, 2015). Dichos procesos han sido liderados por la iglesia y la Educación formal, las que han incidido en la conformación de los futuros ciudadanos desde un enfoque educativo de minorización con carácter monocultural.

En ese contexto, la Educación intercultural está enraizada en un enfoque colonial que se ha desarrollado desde dos vertientes principales: una vinculada a los procesos de inmigración vividos por los países europeos y norteamericanos, quienes se han integrado de forma voluntaria al sistema educativo (DÍAZ-AGUADO, 2007; ESSOMBA, 2014 2014; PRETCEILLE, 2011); la otra, en reconocimiento de la población indígena presente al interior de los países, en distintos continentes (CANDAU, 2010; TUBINO, 2014). Desde este enfoque, los Estados han quitado la autonomía y autodeterminación de los pueblos indígenas, invisibilizando sus formas de organización social y lógica propia de construir conocimiento (TUBINO, 2014; WALSH, 2014).

Desde esa perspectiva, la Educación intercultural en La Araucanía se implementa desde principios del siglo pasado (1925), basada en un enfoque lingüístico mapunzugun-castellano. Este proceso de escolarización trajo consigo la construcción de prejuicios y estereotipos hacia el mapuche (MANSILLA SEPULVEDA; BECERRA PENA; MERINO, 2015). Este hecho está asociado a un desconocimiento histórico de los profesores que se configura en prejuicios y racismo, por lo que se tilda a los niños indígenas como inferiores cognitivamente. Desde este enfoque lingüístico se buscaba avanzar en el proceso de civilización y reproducción del orden hegemónico 
sobre la sociedad mapuche, dirigido sólo a estudiantes indígenas, limitando que estudiantes no indígenas conocieran la riqueza de saberes y conocimientos educativos mapuches.

Desde el enfoque asimilacionista, la Educación intercultural se implementa en la población indígena como una estrategia del Estado para aumentar la "mano de obra bruta", contribuyendo al desarrollo económico del país (SERRANO; PONCE DE LEÓN; RENGIFO, 2012). Así, era necesario que el mapuche se "instruyera" y "civilizara", permitiéndole aportar al desarrollo regional, al ser incorporado a la faena y agricultura (SERRANO; PONCE DE LEÓN; RENGIFO, 2012). Según Bengoa (2008), la inclusión del mapuche a la sociedad chilena fue pensada desde sus inicios como clase obrera y campesina, mediante una integración social básica, sustentada en un empobrecimiento social y cultural. Según Quidel Lincoleo (2015), así se promovía en los niños y jóvenes mapuches el olvido y rechazo a su propia lengua, para su asimilación a la sociedad nacional.

Desde el enfoque de mantención y desarrollo, la Educación intercultural se caracteriza por incorporar la lengua materna y algunas manifestaciones culturales mapuches en los primeros niveles de escolaridad, los cuales no atentaran a los principios y valores universales impuestos por la sociedad dominante (FOERSTER; MONTECINO, 1988). Así se buscaba revertir la deserción escolar y los bajos resultados obtenidos por los niños mapuches en el sistema educativo escolar. El problema de este enfoque es que la Educación intercultural sigue pensada para los mapuches, sin tocar las estructuras del saber y del poder que sustentan el sistema educativo escolar chileno.

Los enfoques actuales de Educación intercultural que constatamos son: 1) el enfoque funcionalista (TUBINO, 2014); y 2) el enfoque crítico (WALSH, 2014). La Educación intercultural desde un enfoque funcionalista, se concibe desde una mirada político-ideológica que se asemeja mucho más a la base epistemológica multiculturalista, promovida por los países anglosajones (LONCÓN, 2016). El problema que aquí observamos es que se invisibilizan los encuentros o desencuentros que surgieron desde el siglo pasado, producto de la relación histórica entre colonizador-colonizado, donde los indígenas asumen el papel de subalterno. Esta realidad de invisibilización de los pueblos indígenas y colectividades subalternizadas es consecuencia de la Educación eurocéntrica occidental con arraigo colonial y un nacionalismo sistémico en América Latina (NOVARO, 2015; NOVARO; PADAWER; BORTON, 2017). 
La Educación intercultural desde un enfoque crítico parte por el cuestionamiento de las estructuras coloniales del poder y del saber, sosteniendo como base que la diferencia se construyó desde el siglo pasado, caracterizado por la jerarquización impuesta por el "blanco" (WALSH, 2014). Desde este argumento, la interculturalidad es un proyecto social, político y ético, que busca avanzar a una Educación intercultural, desde un enfoque decolonial y epistémico. En consecuencia, los antecedentes teóricos nos permiten identificar el problema de invisibilización de "lo indígena" en la Educación escolar, lo cual se refleja en las tensiones epistemológicas que enfrentan en la relación educativa el profesor mentor y el educador tradicional, en la implementación de la EIB. Sostenemos que el educador tradicional está subyugado a la hegemonía del conocimiento occidental representado en la figura del profesor mentor, independiente de la ascendencia étnica de este último.

\section{Relación educativa en la EIB}

La relación educativa es un concepto polisémico y multidimensional, que en este artículo es precisado desde el enfoque psicopedagógico (OUELLET, 2015; POSTIC, 2001). Desde este enfoque, la relación educativa se define como la interdependencia entre el profesor mentor y el educador tradicional, donde se transmiten contenidos educativos objetivos y subjetivos (valores universales), que inciden necesariamente en la formación de persona en la implementación de la EIB (ARIAS-ORTEGA, 2019).

Desde la política pública de EIB, el profesor mentor es definido como el profesional de la Educación escolar encargado de apoyar y monitorear el desenvolvimiento del educador tradicional en la sala de clases, durante el proceso de Enseñanza y aprendizaje del mapunzugun (CHILE, 2011). De acuerdo a los lineamientos normativos del Decreto $n^{\circ} 301$ que reglamenta la calidad de educador tradicional (CHILE, 2018), se establece que éste debe ser elegido por la familia y la comunidad, para que los represente en la Educación escolar de sus niños y jóvenes y, por lo tanto, se incorpore a la escuela para enseñar su lengua y cultura propia (CHILE, 2010; 2018). Lo anterior, puesto que se reconoce en su figura de educador tradicional a una persona sabia, que pone en práctica los valores y principios educativos indígenas para la formación de personas, respondiendo a las demandas de los miembros de la comunidad indígena. Sin embargo, en la práctica observamos que en general los educadores tradicionales no son elegidos por la familia y la comunidad, sino más bien, en general, son asignados discrecionalmente por los directivos de cada establecimiento escolar (ARIAS-ORTEGA, 2019). Incluso, observamos educadores tradicionales que poseen un título de profesor en Educación 
intercultural o bien que es un profesional de la Educación que domina la lengua mapunzugun, por lo que continúan su posición de subalternidad en la relación con el profesor mentor.

Desde un enfoque psicopedagógico, la relación educativa entre el profesor mentor y el educador tradicional, implica: 1) identificar el lugar que ambos agentes educativos ocupan en la sala de clases durante la implementación de la EIB; 2) estudiar cómo actúa el profesor mentor y el educador tradicional de acuerdo a su posición, rol y funciones que asumen en sus intervenciones pedagógicas; 3) analizar las formas de comunicación que surgen entre el profesor mentor y el educador tradicional; y 4) analizar cómo se perciben a sí mismo y al otro durante la implementación de la EIB. En suma, la relación educativa variará respecto de la posición de poder que asume o se le asigna al individuo en la interacción social vertical u horizontal (FISHER, 1991). En el estilo de relación vertical, un sujeto asume el rol de subalterno y, por lo tanto, es el encargado de tener una actitud de pasividad, sometimiento, y actuar de acuerdo a las disposiciones y normas establecidas por el sujeto que asume un rol hegemónico (HENON, 2012), en tanto que, en la relación educativa desde una posición horizontal, existen procesos de acompañamiento entre dos o más individuos, donde se asume una postura simétrica, que implica generar los espacios en los cuales existe una iniciativa continua para preguntar, argumentar e intervenir en las prácticas educativas (HENON, 2012).

\section{Metodología}

La metodología empleada es la investigación educativa desde un enfoque cualitativo, para comprender y explicar las tensiones epistemológicas entre el profesor mentor y el educador tradicional en la implementación de la EIB (ARIAS-ORTEGA, 2019). El enfoque metodológico es exploratorio de tipo descriptivo. Los participantes son 4 profesores mentores y 4 educadores tradicionales, sumando un total de 8 agentes educativos que se desempeñan en escuelas municipales de Educación Básica en el ámbito rural, situadas en comunidades mapuches de la territorialidad Wenteche (personas que habitan la depresión intermedia de La Araucanía), en Padre Las Casas (Figura 1 - ver mapa del territorio donde se desarrolla el estudio). 
Figura 1 - Área de estudio región de La Araucanía, Chile
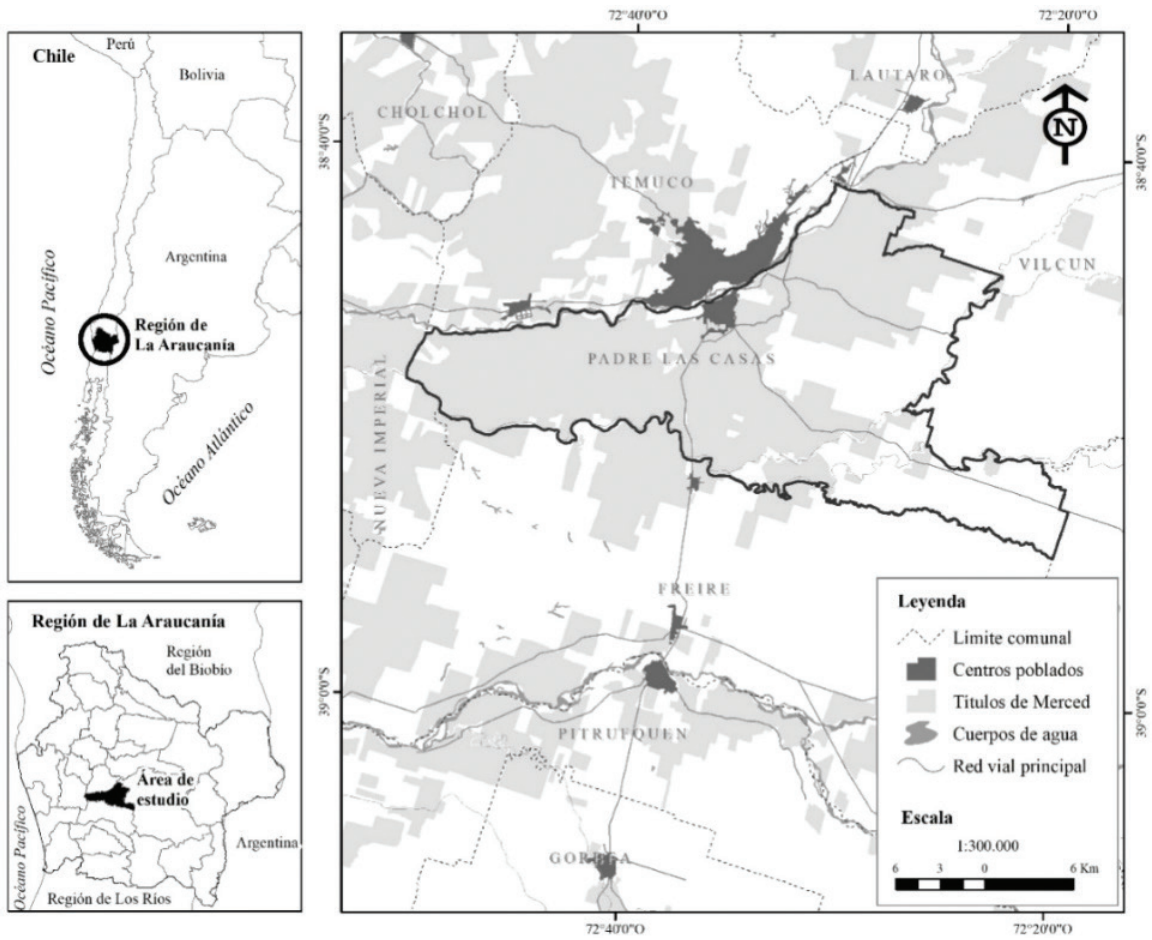

Fuente: Elaboración propia Arias-Ortega (2019)

La técnica que se emplea para la selección de los participantes es intencional, mediante la accesibilidad y representatividad para el investigador (GAUTHIER; BOURGEOIS, 2016). La técnica de recolección de datos e información es la entrevista semi-dirigida que interroga sobre: 1) la relación educativa entre el profesor mentor y educador tradicional; 2) formas en que se incorporan saberes y conocimientos educativos mapuches a la Educación escolar; y 3) racionalidad del conocimiento escolar y del conocimiento educativo mapuche que se incorporan a las prácticas pedagógicas. La técnica de análisis empleada es la teoría fundamentada a través de una codificación abierta y axial (GAUTHIER; BOURGEOIS, 2016). Esta técnica permite la identificación de conceptos, dimensiones e indicadores presentes en el texto de forma explícita o implícita, para construir categorías centrales en relación al objeto de estudio. Con el propósito de identificar las entrevistas de educadores tradicionales y profesores mentores durante su proceso de codificación, se utilizan como nomenclatura los siguientes símbolos: 1) Educador Tradicional (ET), el número de entrevista, escuela Wenteche número 
1 (EW1) y el número [46:46], que corresponde a la ubicación del testimonio en la unidad hermenéutica ${ }^{1}$; y 2) Profesor Mentor (PM), el número de entrevista, escuela Wenteche número 2 (EW2) y el número [125:125], corresponde a la ubicación del testimonio en la unidad hermenéutica.

\section{Resultados}

Las categorías de contenidos centrales que emergen con una mayor recurrencia en el testimonio de los educadores tradicionales y profesores mentores son: 1) relación educativa entre profesor mentor y educador tradicional; y 2) hegemonía del conocimiento escolar.

\subsection{Relación educativa entre el profesor mentor y el educador tradicional}

Esta categoría refiere a la interdependencia que emerge en la interacción social formal e informal en el trabajo colaborativo entre ambos agentes educativos al implementar la EIB. Esta categoría obtiene una frecuencia de 34\%, de un total de 909 recurrencias. En la relación educativa se transmiten contenidos educativos objetivos y subjetivos, que inciden en el trabajo colaborativo entre el profesor mentor y el educador tradicional.

Tabla 1 - Relación educativa entre el profesor mentor y el educador tradicional

\begin{tabular}{lcc}
\hline Códigos & Recurrencia & Porcentaje \\
\hline Vivencias del educador tradicional & 95 & 35,5 \\
Minorización & 91 & 34,0 \\
Relación asimétrica & 81 & 30,3 \\
\hline Total & 267 & $100 \%$ \\
\hline
\end{tabular}

Fuente: Elaboración propia con apoyo del software atlas ti (2019)

De acuerdo a los datos observados en la Tabla 1, la categoría central relación educativa entre el profesor mentor y el educador tradicional se compone de tres subcategorías: vivencias del educador tradicional, minorización y relación asimétrica. Cada una de estas subcategorías está compuesta por códigos que le preceden, los cuales se presentan en la Figura 2.

Precisamos que no es posible dar a conocer el link de la unidad hermenéutica de manera general, producto de los principios y resguardos éticos asumidos con los participantes. Por ello, solo se presentan de manera textual los núcleos de contenidos centrales que emergen del discurso de los participantes. 
Figura 2 - Relación educativa entre el profesor mentor y el educador tradicional

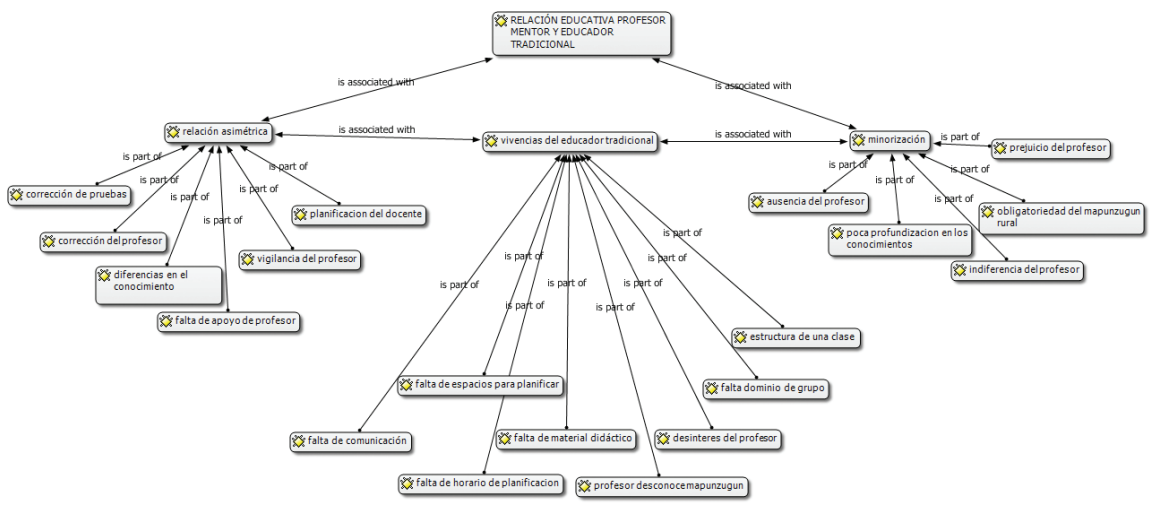

Fuente: Elaboración propia con apoyo del software atlas ti (2019)

La Figura 2 presenta un resumen de las principales características que tiene la relación educativa en la implementación de la EIB. De acuerdo al testimonio de los educadores tradicionales y profesores mentores, la relación educativa que establecen se caracteriza por basarse en una relación asimétrica, donde es el profesor mentor quien adopta una posición de poder en relación al educador tradicional. El primero es quien decide, selecciona, planifica y evalúa las actividades escolares que desarrollan los estudiantes en su proceso educativo, en tanto el educador tradicional asume un rol pasivo, asociado a cumplir las órdenes y mandatos del profesor mentor, constituyéndose de esta manera en su asistente de Educación. De acuerdo al testimonio de los agentes implicados en dicha relación, esta subcategoría obtiene una frecuencia de $30,3 \%$, de un total de 267 recurrencia del corpus analizado.

En la relación asimétrica, las principales características están dadas por los siguientes códigos, falta de apoyo del profesor en el aula. Esto se expresa en el siguiente testimonio: "a mí la profesora me dejaba sola en la sala, entonces no había un acompañamiento del profesor mentor, no me decían [que] vamos a pasar esto, o vamos a planificar ${ }^{2}$ [juntas]" (ET1EW1 [46:46]). Ella no se comunicaba conmigo para decirme "usted va a hacer esto, yo hago esto otro, yo escribo, pasamos láminas, pasemos [a los estudiantes] papel de oficio [para] que

\footnotetext{
Precisamos que los códigos que ilustran la presentación de los resultados están referidos principalmente a educadores tradicionales, puesto que de los 4 profesores mentores que participaron de la entrevista semi-dirigida, solamente dos respondieron a los contenidos interrogados. Este se debe a que los agentes educativos que dominan los saberes y conocimientos mapuches interrogados, son dominados principalmente por los educadores tradicionales.
} 
dibujen. Nada me decía, es como si no estuviera [en la sala]" (ET1EW1 [46:46]). De acuerdo al testimonio se observan procesos de invisibilización del educador tradicional en el aula y en la escuela en general.

En relación a las diferencias del conocimiento y la corrección del profesor hacia el educador tradicional durante sus intervenciones pedagógicas, generan situaciones de tensión y roces entre ambos. De acuerdo al testimonio de los educadores tradicionales, el conocimiento que posee el profesor se impone en el desarrollo de las actividades escolares como una verdad absoluta. Lo anterior, queda reflejado en el siguiente testimonio:

me corrige cuando hay un error, entre los dos nos vamos enseñando, él me enseña a pronunciar bien algunas palabras en castellano, yo siento admiración por el profesor porque él estudió para ser profesor. Pero eso debiera ser todo lo contrario, porque a veces yo tengo más riqueza de conocimiento de mi cultura, y eso que yo no estudié (ET2EW2[72:72]).

Otro elemento que da cuenta de esta relación asimétrica, está dado por los procesos de planificación de las actividades escolares, donde el profesor mentor es quien de manera individual planifica y selecciona los contenidos educativos mapuches, para ser incorporados en la intervención pedagógica. Junto con ello, niega al educador tradicional la posibilidad de plantear los contenidos educativos mapuches pertinentes de incorporar, desde un método educativo propio. Al respecto un educador tradicional señala que: "ella dirige la clase, me dice, por ejemplo, vamos a hacer esta clase, vamos a pasar esta página y esta otra página" (ET3EW3 [67:67]). Lo anterior da cuenta de la supremacía de los métodos educativos escolares que son característicos durante la implementación de la EIB y que no necesariamente responden a las prácticas epistémicas para educar a los niños y jóvenes mapuches. Además, se niega al educador tradicional participar en el trabajo pedagógico, y junto con ello, se le quita la posibilidad de cumplir con el rol educativo otorgado por la comunidad: ser el mediador entre la familia-comunidad-escuela en la formación intercultural de los estudiantes. Es así como en la práctica se impone el rol otorgado al educador tradicional por aspectos normativos, que lo habilitan como la persona encargada de transmitir los saberes y conocimientos educativos mapuches en la institución escolar.

En relación a la subcategoría vivencias del educador tradicional, obtiene una frecuencia de $35,5 \%$, y refiere a las experiencias o sucesos que ha vivido en 
su calidad de educador tradicional en la institución escolar. En la dimensión metodológica, observamos experiencias negativas en la escuela, en relación al desinterés que manifiestan los profesores respecto a la incorporación de los saberes y conocimientos educativos mapuches al aula. Es así como emerge la dificultad de desarrollar un trabajo en conjunto entre profesor mentor y educador tradicional, debido al desconocimiento de ambos actores educativos, respecto del mapunzugun, lo que dificulta el desarrollo de un trabajo colaborativo. Tal como se manifiesta en el siguiente testimonio: "todos los profesores usan puro español, si no saben el mapunzugun, nadie sabe, nadie ningún profesor" (ET2EW2 [263:263]). En relación al desinterés del profesor, un educador tradicional menciona: "los profesores dicen que los conocimientos mapuches no les van a servir [a los estudiantes] en el futuro, eso dicen, [se preguntan] para qué va a servir el mapunzugun, si los niños no le van a sacar ningún provecho" (ET2EW2[229:229]).

En relación a la dimensión didáctica, las principales vivencias del educador tradicional están asociadas a la falta de material didáctico para el trabajo con los estudiantes. También, observamos que el profesor mentor niega el trabajo educativo fuera del aula como un espacio de aprendizaje que se ajuste a la didáctica del conocimiento educativo mapuche. Adicionalmente, los educadores tradicionales no cuentan con métodos educativos propios sistematizados. Al preguntar si esto dificulta el trabajo educativo, un educador tradicional señala: "sí incide, porque la lana por ejemplo cuando hay que enseñarles el pülalkantun (juego con hilos) a los niños, yo no tengo lana aquí en la escuela y tengo que traer la lana de mi casa" (ET2EW2 [101:101]). En la dimensión pedagógica, las principales vivencias del educador tradicional se caracterizan por su obligatoriedad de aprender el lenguaje pedagógico, comprender la estructura de una clase, así como la falta de horario para planificar las clases en conjunto con el profesor mentor. Al respecto, un educador tradicional señala: "antes teníamos horas para planificar en conjunto, ahora no. Yo tengo mi tiempo libre de una hora para planificar, pero la profesora no tiene tiempo libre, en ese tiempo ella está en clases. Entonces, ¿cómo vamos a planificar [en conjunto]?” (ET2EW2 [85:85]).

De acuerdo a los testimonios de los educadores tradicionales constatamos la necesidad de revisar la configuración de los procesos educativos escolares, con la intención de reestructurar estas prácticas pedagógicas y revertir las relaciones asimétricas entre profesor mentor y educador tradicional.

En relación a la subcategoría minorización, esta obtiene una frecuencia de $34 \%$, asociado a las vivencias del educador tradicional en la relación que 
establece con el profesor mentor. Los educadores tradicionales atribuyen sus propios significados a las acciones o situaciones en los cuales ellos se sienten minorizados en las prácticas educativas. Asimismo, se observa poca profundización en los conocimientos indígenas por parte del profesor, por considerarlos de menos valor para la Educación, para el desarrollo social y económico de los estudiantes. Al respecto, un educador tradicional señala: "porque como le decía, la gente dice siempre que no le va a servir mucho el mapuche kimün, (conocimiento mapuche) el mapunzugun" (ET2EW2[239:239]). Según el testimonio de educadores tradicionales, también han vivido situaciones de prejuicios de los profesores hacia ellos o hacia sus estudiantes en tanto mapuches:

la pena que tengo, es que siempre hay muchas diferencias entre las personas, $[\ldots]$ yo he tenido que defender tantas veces mi postura, un día en una reunión les dije, ustedes dicen mapuche, indio, pero saben, yo tengo lo mismo que tienen ustedes, tengo los mismos ojos, respiro por ahí mismo, tengo mis orejas, tengo mi pelo, excepto que soy de una tez morena, pero tengo la misma lengua como hablan ustedes, no entiendo cuál es la diferencia (ET4EW4[202:202]).

Los testimonios de los educadores tradicionales dan cuenta de una relación tensionada constantemente, en donde son ellos quienes deben asumir una posición de pasividad y sumisión dentro de la institución escolar, debido a la hegemonía del conocimiento escolar y la supremacía de la sociedad eurocéntrica occidental.

\subsection{Hegemonía del conocimiento escolar}

La categoría hegemonía del conocimiento escolar refiere a la transmisión de los saberes y conocimientos educativos de corte eurocéntrico occidental que prevalecen en los procesos educativos institucionalizados en la escuela como únicos y válidos para la Enseñanza y el aprendizaje, que se transmiten con base en métodos educativos escolares. Dicha categoría obtiene una frecuencia de 16,5\% de un total de 909 recurrencias en el testimonio de los profesores mentores y educadores tradicionales. Así, en la Figura 3 se presenta la categoría asociada a 2 subcategorías: 1) método educativo escolar que refiere a las formas de transmitir los contenidos educativos que históricamente han predominado en la institución escolar; y 2) evaluación monocultural que refiere a los procesos evaluativos que son llevados a cabo en la escuela y que se configuran desde una perspectiva positivista, según la racionalidad eurocéntrica occidental. Ambas subcategorías obtienen las siguientes recurrencias. 
Tabla 2 - Hegemonía del conocimiento escolar

\begin{tabular}{lcc}
\hline Códigos & Recurrencia & Porcentaje \\
\hline Método educativo escolar & 81 & $63,7 \%$ \\
Evaluación monocultural & 46 & $36,2 \%$ \\
\hline Total & 127 & $100 \%$ \\
\hline
\end{tabular}

Fuente: Elaboración propia con apoyo del software atlas ti (2019)

Según los datos que se observan en la Tabla 2, la subcategoría método educativo escolar obtiene una frecuencia de $63,7 \%$ de un total de 127 recurrencias y se compone de los siguientes códigos: memorización, traducción mapunzugun-castellano, trabajo con láminas, libros de estudio, debate, dibujos, Enseñanza de vocabulario, en donde prevalece el monolingüismo del castellano, en tanto la subcategoría evaluación monocultural obtiene una frecuencia de $36,3 \%$ y está compuesta por los siguientes códigos: trabajos escritos, evaluación de la oralidad, pruebas, guías de aprendizaje, disertaciones y evaluación de la comprensión lectora, según la estructura gramatical de la lengua española.

Figura 3 - Hegemonía del conocimiento escolar

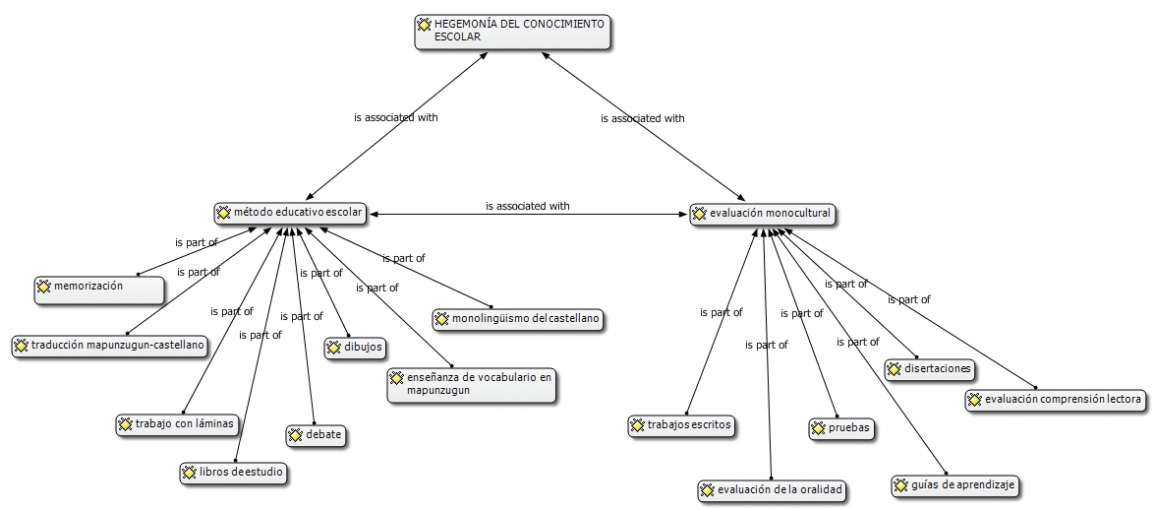

Fuente: Elaboración propia con apoyo del software atlas ti (2019)

La Figura 3, presenta un resumen de los elementos develados en el testimonio de los profesores mentores y educadores tradicionales en relación a la implementación de la Educación intercultural. En ella se puede observar que los métodos educativos predominantes durante el proceso de incorporación de los saberes y conocimientos 
educativos mapuches continúan arraigados a las prácticas hegemónicas de la escuela y de la Educación escolar. Se releva la memorización en la cual se espera que los niños reproduzcan de manera literal los contenidos educativos incorporados, sin generar procesos de comprensión, reflexión y apropiación de dichos contenidos, desde su propio marco de referencia. Al respecto, un testimonio señala: "los niños aprenden los contenidos, reproducen más que nada, los repiten y memorizan cada palabra" (ET3EW3 [97:97]). Esta lógica de trabajo es reiterativa en los establecimientos, en la cual se asume que en la medida que el estudiante memorice, mayor conocimiento tendrá. Un testimonio señala: "claro, lo que pasa es que vamos contabilizando cuántas palabras dicen buenas y malas, para saber cuánto conocimiento le quedó en la memoria" (ET1EW1 [85:85]). Ambos testimonios dan cuenta de una descontextualización de la Enseñanza y aprendizaje a los métodos educativos mapuches para la incorporación de los saberes y conocimientos educativos propios. En relación a la incorporación de la lengua mapunzugun a la escuela, el testimonio de los educadores tradicionales da cuenta de la constante traducción mapunzugun-castellano característico de la asignatura de lengua indígena, tal como se observa en el siguiente testimonio:

yo les cuento el epew todo en mapunzugun, todas las conversaciones que hago con los niños son en mapunzugun, después se los redacto en castellano [...]. no saco nada con decirle esto es aquí o esto es acá en mapunzugun y luego irme, porque me voy y dejo al niño aquí simplemente, dando vote como pelota, porque no entienden nada (ET3EW3 [75:75]).

De acuerdo al testimonio podríamos inferir que esta metodología de trabajo ha sido adoptaba para facilitar el proceso de Enseñanza y aprendizaje del mapunzugun en la escuela. En relación a las actividades y materiales que utilizan los estudiantes, predominan las láminas de trabajo, el apoyo del libro de estudio, los debates y la apropiación de vocabulario en mapunzugun, asociado a colores, números y el monolingüismo del castellano. Al respecto, los testimonios señalan: "formaban frases mirando láminas en mapunzugun [...] le coloqué varios conceptos en mapunzugun con su nombre y al otro lado un número, entonces ellos tenían que unir ambos globos y así formar frases abajo" (PM2EW2 [418:418]). Otro testimonio señala: "todos los profesores usan puro español, si no saben el mapunzugun, nadie sabe, nadie ningún profesor" (ET2EW2 [263:263]).

En relación a la subcategoría evaluación monocultural, se evidencia que sigue una constante de cuantificar los conocimientos que adquieren los estudiantes como una forma de dar cuenta de los contenidos educativos incorporados. A su vez, 
la evaluación se constituye en la forma de cumplir y controlar en relación a la normativa impuesta por el Mineduc, referida a la necesidad de obtener una calificación para medir los aprendizajes escolares, que "supuestamente" adquieren los estudiantes, tal como se observa en el siguiente testimonio: "el proceso de evaluación es obligatorio y se realiza en forma escrita en pruebas en español" (PM4EW4 [15:125]). Lo anterior es reafirmado por el siguiente testimonio: "sí los contenidos van con nota [...]. Para evaluar a veces le hacemos preguntas orales, yo hago la pregunta oral y la profesora va a anotando quién contestó mejor y quién no. También usamos guías de trabajo y pruebas" (ET2EW2 [81:81]). Así, es posible evidenciar que la evaluación es monocultural eurocéntrica occidental, aplicada a los niños de forma subjetiva y que no evalúa aprendizajes adquiridos, sino más bien la capacidad de memorización.

\section{Discusión y conclusiones}

La discusión de la literatura normativa y científica a nivel nacional e internacional (QUINTRIQUEO; QUILAQUEO, 2019; RUANO; MORILLO; GONZALEZ, 2018; WALSH, 2014) nos permite sostener la necesidad de una Educación intercultural que considere los conocimientos indígenas para contextualizar la formación de estudiantes y futuros profesionales desde enfoques transdisciplinarios, para mejorar la relación educativa entre los agentes del medio educativo y social, superando de esta manera el discurso retórico sobre Educación intercultural (NOVARO, 2015). Asimismo, los estudios en contextos de colonización remarcan la necesidad de incorporar prácticas epistémicas propias de los pueblos indígenas para sustentar una Educación escolar intercultural, mediante la sensibilización, la planificación e implementación de una política pública intercultural (BISHOP, 2010; RUANO, MORILLO; GONZALEZ, 2018). Esto plantea el desafío de relevar la forma de indagar, conocer, comprender y explicar la construcción del orden del conocimiento indígena, según la memoria social y las ontologías relacionales, donde la relación del hombre con el medioambiente va más allá de lo humano, expresado en una visión holística y mística del mundo (CAJETE, 1994; ESCOBAR, 2014). Existen evidencias científicas sobre la pertinencia de profundizar en la episteme indígena para democratizar y contextualizar la Educación intercultural para todos, que implique a los actores del medio educativo y social desde un pluralismo epistemológico intercultural (MORILLO; AVILES; UYAGUARI, 2019; RUBIO; OSANDON; QUINTEROS, 2019).

En ese contexto, los resultados de investigación en el marco de la implementación de la EIB nos permiten sostener que la relación educativa entre profesor mentor y educador tradicional se caracteriza por permanentes desencuentros. Constatamos que ello se debe a los siguientes aspectos: 1) la invisibilización 
de los educadores tradicionales en el aula, en la implementación de la EIB; 2) el control sistemático que establece el profesor mentor sobre los espacios educativos, los dominios didácticos, pedagógicos, respecto del orden del conocimiento escolar eurocéntrico occidental; 3) la imposibilidad del educador tradicional para cumplir con el rol social y político que le encarga la familia y la comunidad; 4) el desconocimiento de los saberes y conocimiento educativo mapuche, por parte del profesor mentor, pero también del educador tradicional; 5) la imposibilidad del trabajo colaborativo entre el profesor mentor, el educador tradicional y los sabios de la comunidad indígena; 6) la desvalorización tanto del conocimiento educativo como de la lengua mapunzugun en la Educación escolar; 7) la normalización de la lengua mapunzugun y de los conocimientos educativos mapuches a la estructura epistémica del conocimiento escolar; y 8) la predominancia de un sistema de evaluación de la Enseñanza y del aprendizaje basado en la producción y el control, lo que coarta la emancipación de los estudiantes desde sus respectivos marcos sociales y culturales. Lo anterior se sustenta en la institucionalidad de la Educación escolar y la política pública en Chile sobre Educación intercultural, en las que se les niega a los educadores tradicionales la posibilidad de calificar el aprendizaje escolar de los estudiantes. Este hecho incide en las tensiones que emergen en la relación educativa, caracterizándola por las estructuras de poder colonial arraigado en las prácticas educativas escolares en contextos de colonización, lo que se expresa en una imposición hegemónica de la evaluación monocultural eurocéntrica occidental de los estudiantes. En esta perspectiva, se limitan las posibilidades de incorporar el conocimiento mapuche sobre la didáctica y pedagogía indígena, lo que permitiría generar una relación educativa simétrica entre el profesor mentor y el educador tradicional en la implementación de la EIB.

Estudios realizados sobre la implementación de la EIB en La Araucanía han constatado resultados similares al nuestro, poniendo en evidencia prácticas pedagógicas entre el profesor mentor y el educador tradicional, basadas en la traducción del castellano al mapunzugun y viceversa (LONCON, 2016; QUIDEL CATRILAF, 2011). Asimismo, se utilizan preguntas y respuestas para orientar el proceso educativo en el aula, utilizando los recursos didácticos normativos disponibles en la institución escolar, sin posibilidad de recurrir a métodos y estrategias educativas mapuches. Aunque se observa indagación en el medio familiar y comunitario, son situaciones de aprendizaje fundamentadas en el orden del conocimiento eurocéntrico occidental, particularmente en la lectura y la escritura (LONCON, 2016; QUIDEL CATRILAF, 2011). Es así como la Educación intercultural queda en el plano normativo y en la retórica del discurso oficial que transmite 'la efectividad' de esta política, desde el poder central, de quienes están a cargo de la gestión educativa (sostenedores, directores de escuela, encargados 
de la unidad técnica pedagógica) y de quienes la concretizan en el aula como es el caso de los profesores mentores y educadores tradicionales.

En conclusión, el estudio devela la necesidad de co-construir la Educación intercultural en el contexto indígena, fundamentado desde un pluralismo epistemológico intercultural con la implicación de los actores del medio educativo y social. En esta perspectiva, proponemos descolonizar el saber escolar como único y valido para la humanidad. Así, la Educación intercultural debe ser un enfoque que sustente acciones educativas centradas en el aprendizaje, teniendo presentes actividades que favorezcan el desarrollo de valores, actitudes y comportamientos orientados a combatir el racismo, los estereotipos y prejuicios. Asimismo, es necesario generar procesos educativos desde un pluralismo epistemológico que permita generar un diálogo de saberes desde los actores del medio educativo y social. 


\title{
Epistemological tensions in the implementation of Bilingual Intercultural Education
}

\begin{abstract}
The article seeks to investigate the existing epistemological tensions between the mentor teacher and traditional educator (Mapuche sage), in the implementation of Bilingual Intercultural Education (EIB) in three schools in La Araucania, Chile. The methodology used is qualitative research, through the application of semi-interviews directed to mentor teachers and traditional educators in charge of implementing the IBE. The main results show that there is a colonial educational relationship between the mentor teacher and the traditional educator, which limits the implementation of IBE. These tensions are associated with practices of minorization, asymmetry and hegemony of Western Eurocentric rooted school knowledge on the Teaching of the Mapuche language and culture.
\end{abstract}

Keywords: Educational Relationship. Colonial education. Intercultural education.

\section{Tensões epistemológicas na implementação da Educação Intercultural Bilíngue}

\section{Resumo}

O artigo procura investigar as tensões epistemológicas existentes entre o professor mentor e o educador tradicional (sábio Mapuche), na implementação da Educação Intercultural Bilingue (EIB) em três escolas de La Araucanía, Chile. A metodologia utilizada foi a pesquisa qualitativa, através da aplicação de semientrevistas direcionadas aos professores orientadores e aos educadores tradicionais encarregados da implementação do EIB. Os principais resultados mostram que existe uma relação educacional colonial entre o professor mentor e o educador tradicional, o que limita a implementação do EIB. Essas tensões estão associadas às práticas de memorização, assimetria e hegemonia do conhecimento da escola eurocêntrica ocidental sobre o ensino da língua e da cultura mapuche.

Palavras-chave: Relação educacional. Educação colonial. Educação intercultural. 


\section{Referencias}

ARIAS-ORTEGA, K. Relación educativa entre el profesor mentor y el educador tradicional en la educación intercultural. Tesis (Doctoral en Educación) - Universidad Católica de Temuco, Chile, 2019.

BENGOA, J. Historia del pueblo mapuche siglos XIX y XX. Santiago: LOM Ediciones, 2008.

BISHOP, R. Effective teaching for indigenous and minoritized students. Procedia Social and Behavioral Sciences, Waikato, v. 7, p. 57-62, 2010. https://doi.org/10.1016/j.sbspro.2010.10.009

CAJETE, G. Look to the mountain: an ecology of Indigenous education. Skyland: Kivaki, 1994.

CANDAU, V. M. F. Educación intercultural en América Latina: distintas concepciones y tensiones actuales. Estudios Pedagógicos (Vadivia), v. 36, n. 2, p. 333-342, Jan. 2010.

CHILE. Ministerio de Educación. Consulta a los Pueblos Indígenas. Informe de resultados: función docente del educador(a) tradicional para la implementación del sector de lengua indígena. Santiago: Programa de Educación Intercultural Bilingüe, 2011.

CHILE. Ministerio de Educación. Informe sobre educación intercultural bilingüe. Santiago de Chile: Programa de Educación Intercultural Bilingüe, 2018

CHILE. Ministerio de Educación. Orientaciones Programa de Educación Intercultural Bilingüe. Santiago: Departamento Superior de Educación General, 2010.

DÍAZ-AGUADO, M. Educación intercultural y aprendizaje cooperativo. Madrid: Edicionespirámide, 2007.

DIETZ, G.; MATEOS CORTÉS, L. S. Interculturalidad y educación intercultural en México: un análisis de los discursos nacionales e internacionales en su impacto en los modelos educativos mexicanos. México, DF: Secretaría de Educación Pública Coordinación General de Educación Intercultural y Bilingüe, 2011.

ESCOBAR, A. Sentipensar con la tierra: nuevas lecturas sobre desarrollo, territorio y diferencia. Medellín: Universidad Autónoma Latinoamericana, 2014 
ESSOMBA, M. A. Diversity of contexts, unity of purposes: preparing for successful school achievement by those children and youngsters in Europe from migrant background. Citizenship Teaching \& Learning, [s. 1.], v. 9, n. 2, p. 117-133, May 2014. https://doi.org/10.1386/ctl.9.2.117_1

FISHER, G. N. Les concepts fondamentaux de la psychologie sociale. Paris: Dunod. 1991.

FOERSTER, R.; MONTECINOS, S. Organizaciones, líderes y contiendas mapuches (1900-1970). Santiago: CEM, 1988.

GAUTHIER, B.; BOURGEOIS, I. Recherche sociale de la problématique à la collecte des données. Québec: Presses de l'Université du Québec, 2016.

HENON, S. Percevoir, comprendre et analyser la relation éducative: identification de schèmes d'action y transformation de l'habitus relationnel. Paris : UFR Sciences de l'Homme et de la Société, 2012.

LONCÓN, E. Consultoría que oriente el proceso de definición del concepto de interculturalidad para el sistema educativo. Santiago: Programa Educación Intercultural Bilingüe del Ministerio de Educación, 2016.

MANSILLA SEPULVEDA, J.; BECERRA PENA, S.; MERINO, M. E. Curriculum violence occidental knowledge hegemony in relation to indigenous knowledge. Procedia - Social and Behavioral Sciences, Amsterdam, v. 190, p. 434-439, May 2015. https://doi.org/10.1016/j.sbspro.2015.05.022

MORILLO, M. M.; AVILES, E. P. C.; UYAGUARI, J. La trascendencia del sumak kawsay para nuestra comprensión de la educación, la ética y la política. Ensaio: Avaliação e Políticas Públicas em Educação, Rio de Janeiro, v. 28, n. 106, p. 241-264, jan./mar. 2019.

NOVARO, G. ¿Desafíos interculturales o una interculturalidad desafiada? Experiencias en escuelas con población migrante boliviana en Argentina. Temas de Educación, La Serena, v. 21, n. 2, p. 381-390, 2015.

NOVARO, G.; PADAWER, A.; BORTON, L. Interculturalidad y educación en Argentina desde una Perspectiva Comparativa. Educação \& Realidade, Porto Alegre, v. 42, n. 3, p. 939-958, 2017.

OUELLET, S. Relations éducatives et apprentissage: regards diversifiés de professionnels en éducation et futurs chercheurs. Québec: Presses de 1’Université du Québec, 2015. 
PALADINO, M.; CZARNY, G. Povos indígenas e escolarização: discussões para repensar novas epistemes nas sociedades latino-americanas. Rio de Janeiro: Garamond, 2012.

POSTIC, M. La relation éducative. 6. ed. Paris : Presses Universitaires de France, 2001.

PRETCEILLE, M. A. La pedagogie interculturelle: entre multiculturalisme et universalisme. Lingvarum Arena, Porto, v. 2, p. 91-101, 2011.

QUIDEL CATRILAF, G. Estrategias de enseñanza de la lengua mapunzugun en el marco del PEIB Mineduc-Orígenes (Comuna de Padre las Casas, IX Región-Chile). Cuadernos Interculturales, Viña del Mar, v. 9, n. 16, p. 61-80, primer semestre 2011.

QUIDEL LINCOLEO, J. Chumgelu ka chumgechi pu mapuche ñi kuxankapagepan ka hotukagepan ñi rakizuam ka ñi püjü zugu mew. In: Antileo, E., et al. (eds.). Awükan ka kuxank na zugu Wajmapu mew: violencias coloniales en Wallmapu. Temuco: Comunidad Historia Mapuche, 2015. p. 21-56.

QUINTRIQUEO, S.; ARIAS-ORTEGA, K. Educación Intercultural articulada a la episteme indígena en Latinoamérica: el caso Mapuche en Chile. Diálogo Andino, Arica, n. 59, p. 81-91, jun. 2019. http://dx.doi.org/10.4067/S0719-26812019000200081

QUINTRIQUEO, S.; QUILAQUEO, D. Educación e interculturalidad: aproximación crítica y decolonial en contexto indígena. Temuco: Universidad Católica de Temuco, 2019.

RUANO, J. C.; MORILLO, M. M.; GONZÁLEZ, F. J. A. Educación transdisciplinar: formando en competencias para el buen vivir. Ensaio: Avaliação e Políticas Públicas em Educação, Rio de Janeiro, v. 26, n. 100, p. 619-644, jul./set. 2018. https://doi.org/10.1590/s0104-40362018002601487

RUBIO, G.; OSANDON, L.; QUINTEROS, F. La experimentación pedagógica territorial y la democratización del sistema educativo. Lecciones del Plan Experimental de Educación Rural de San Carlos (1944-1947). Ensaio: Avaliação e Políticas Públicas em Educação, Rio de Janeiro, v. 27, n. 102, p. 88-107, jan./mar. 2019. https://doi.org/10.1590/s0104-40362018002701488 
SERRANO, S.; PONCE DE LEÓN, M.; RENGIFO, F. Historia de la Educación en Chile. Santiago: Taurus, 2012. Tomo 1: Aprender a leer y escribir (1810-2010).

TUBINO, F. El trasfondo epistémico de los conflictos interculturales. Guadalajara, v. 6, n. 11, p. 1-6, jul./dic. 2014.

TURRA DÍAZ, O. Tensiones en la enseñanza y aprendizaje de la historia en contexto interétnico: significaciones y experiencias escolares de jóvenes pehuenches. Atenea, Concepción, v. 513, p. 263-278, I sem. 2016. https://doi.org/10.4067/S0718-04622016000100017

VALDEBENITO G., X. Hacia una comprensión del vínculo entre las prácticas de enseñanza de educadores y educadoras tradicionales indígenas y el espacio escolar. Santiago: Ministerio de Educación de Chile, 2017.

WALSH, C. Lo pedagógico y lo decolonial: entretejiendo caminos. [S. 1.]: Colectivo Zapatandole al mal gobierno, 2014.

\section{Información del autores}

Katerin Arias-Ortega: Doctora en Ciencias de la Educación. Académica del Departamento de Psicología de la Facultad de Ciencias de la Salud. Universidad Católica de Temuco. Contato:karias@uct.cl

iD http://orcid.org/0000-0001-8099-0670

Segundo Quintriqueo: Doctor en Ciencias de la Educación. Profesor Titular del Departamento de Ciencias de la Educación de la Universidad Católica de Temuco. Contato: squintri@uct.cl

iD http://orcid.org/0000-0002-7228-4095 